Proceedings of the IX International Conference ION 2012, Kazimierz Dolny, Poland, June 25-28, 2012

\title{
Surface Morphological Properties of Mo-Based Thin Films on Glass
}

\author{
I. TAShlykov ${ }^{a}$, A. Turavets ${ }^{a}$ AND P. ZuKOWski ${ }^{b, *}$ \\ ${ }^{a}$ Belarusian State Pedagogical University, Sovetskaya 18, 220050 Minsk, Belarus \\ ${ }^{b}$ Lublin University of Technology, Nadbystrzycka 38d, 20-618 Lublin, Poland
}

\begin{abstract}
In that report we observe the initial stages in the process of film growth at different irradiation doses. Investigations of influence of Mo deposition on glass substrates by means of self-ion-assisted deposition on its topography (atomic force microscopy) and wettability (sessile-drop method) were carried out. It was found out that with an increase of the irradiation dose, the average roughness and the contact angle increases rapidly at first and then decrease. 2.45-2.77 increase in the contact angle of water when Mo-based coating was deposited on the glass was observed.
\end{abstract}

DOI: $10.12693 /$ APhysPolA.123.840

PACS: 88.40.jm, 68.08.Bc, 68.37.Ps

\section{Introduction}

Manufacturing of solar cells consists of a series of steps. The solar cell basic structure is as follows: (1) back contact; (2) semiconductor layer (CIGS); (3) top contact including a $\mathrm{ZnO}$ layer. In addition to these functional layers, the cell is dependent on a glass substrate to support it mechanically. Several metals have been investigated to be used as back contact. The choice of molybdenum (Mo) is based on the requirements imposed by application and processing steps. Mo has desired stability at the processing temperature, resistance to alloying with the absorber layer elements, and low contact resistance to the absorber layer. The thin Mo layer also is considered as crystal seed grains for the growth of absorber [1].

Vacuum evaporation is relatively successful in the cases when improved adhesion is important [2]. The self-ion-assisted deposition (SIAD) process has some unique properties. For example amount of average deposited energy density in the atomic collision cascade is several times higher compared with ther ion-beam-assisted deposition process [3]. The original SIAD method provides ion-beam-mixing of the substrate atoms and those of a thin film without introducing an admixture of noble gases (SIAD) and allows to create a gradual transition between the deposited film and the substrate, and to deposit films with less built-in strain. The advantage of this method is the use of ion species which are the same as the coating material. These properties can result in films with a more durable bond to the substrate. SIAD was known to affect crystalline structure, packing density, adhesion and surface smoothness of the film and thus improving the quality of thin film for functional devices [4].

The Mo thin films have been deposited on glass substrates by SIAD for the first time. This work is only the

*corresponding author; e-mail: pawel@elektron.pol.lublin.pl first stage of the research devoted to the preparation of the back contact solar cell by SIAD. In the first stage of our research we investigated the initial stages in the process of film growth at different irradiation doses before development of a continuous structure, influence of the irradiation doses during Mo deposition on the glass substrates on its topography and wettability.

\section{Experimental}

SIAD experiments were performed using a resonance vacuum arc ion source [4]. Film deposition was carried out in a vacuum chamber with a pressure of $10^{-2} \mathrm{~Pa}$. Substrate plates were floated to a negative potential with respect to the ion source of $10 \mathrm{keV}$. The beam current density was kept at $5.1 \mu \mathrm{A} / \mathrm{cm}^{2}$. The irradiation dose $D$ of Mo ions was $1.2 \times 10^{16}, 3.2 \times 10^{16}, 5.2 \times 10^{16}, 8.1 \times 10^{16}$, $1.1 \times 10^{17} \mathrm{ions} / \mathrm{cm}^{2}$.

The surface morphology of the samples was investigated by means of atomic force microscope "NT-206" using cantilevers CSC21. Atomic force microscopy (AFM) images were analyzed using the program "SurfaceXplorer 1.3.11". Lateral force mode of AFM allows us to distinguish areas with different frictional properties as well as to obtain edge-enhanced images of any surface. The cantilever torsion $(Z)$ is measured by the microscope optical recording system and presented in arbitrary units. The spatial distribution of lateral forces within the area of $10 \times 10 \mu \mathrm{m}^{2}$ was characterized by the mean value $\left(Z_{\text {mean }}\right)$.

The wetting behaviour is characterized by the value of the contact angle $(\mathrm{CA}, \Theta)$. CA measurements were based on the sessile-drop method. The original setup for measuring the value of the $\mathrm{CA}$ is demonstrated in Fig. 1. The wetting agent was doubly distilled water (volume $\approx 15 \mu \mathrm{l}$ ). The drop is placed on the surface of a sample with the help of syringe 3 . The stepper motors and control unit 5 control the positioning of the sample and extrusion of the drop. CA measurements were carried out $60 \mathrm{~s}$ after the droplet was deposited on the film. The 


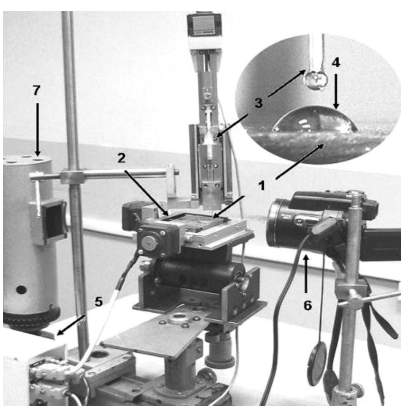

Fig. 1. Equipment for contact angle measurements: 1 - sample, 2 - device of positioning of the sample, 3 - syringe, 4 - water drop, 5 - block of electronics, 6 - digital camera, 7 - source of monochromatic light.

digital camera 6 is connected to a computer and has remote shutter release.

$\mathrm{CA}$ is measured automatically, thus eliminating operator intervention. A specially designed program "Angle" filters the image of the system "water drop-substrate-air" so that the gas phase was selected and the image of the system was projected onto a plane. The operator visually determines the point of three-phase contact on the image, then the program automatically builds a line of the sample surface, and a tangent to the droplet surface, and measures the angle between them. The total error of our measurements is $0.5^{\circ}$.

\section{Results and discussion}

\subsection{Surface morphology}

The AFM was utilized to examine the topography and the roughness of the initial glass and Mo thin films on glass. The images are shown in Fig. 2. The topography parameters are listed in Table.

TABLE

The topography parameters and significances of the CA of the investigated samples.

\begin{tabular}{l|c|c|c|c|c|c}
\hline \hline \multicolumn{2}{l|}{$D\left[10^{16}\right.$ ions $\left./ \mathrm{cm}^{2}\right]$} & 1.2 & 3.2 & 5.2 & 8.1 & 11 \\
\hline$R_{\text {a }}[\mathrm{nm}]$ & 2.2 & 1.8 & 2.0 & 2.7 & 2.3 & 2.3 \\
$Z_{\text {mean }}$ & 221.7 & 245.9 & 280.6 & 273.3 & 282.0 & 279.8 \\
$\mathrm{CF}$ & & 56.9 & 33.6 & 34.5 & 48.1 & 96.5 \\
$\Delta h[\mathrm{~nm}]$ & & 7.2 & 6.0 & 4.6 & 3.0 & 3.0 \\
$\Theta\left[{ }^{\circ}\right]$ & 25.1 & 62.8 & 67.4 & 69.7 & 60.8 & 56.7
\end{tabular}

As can be seen, the irradiation doses used in this experiment do not allow to obtain a uniform and continuous coating. The images present the initial stage of formation and growth of the films. The degree of homogeneity coating and its thickness by building histograms of heights and cross-sections of 2D AFM images of the topography on the most common heights can be found.

Assessing the $\%$ of coating formation $(\mathrm{CF})$, we see a decline from $56.9 \%$ to $33.6 \%$ in the initial stage of the coating formation. This is possible only when the observed multi-level surface is not one of the coating and substrate, but that of the coating deposited unevenly on

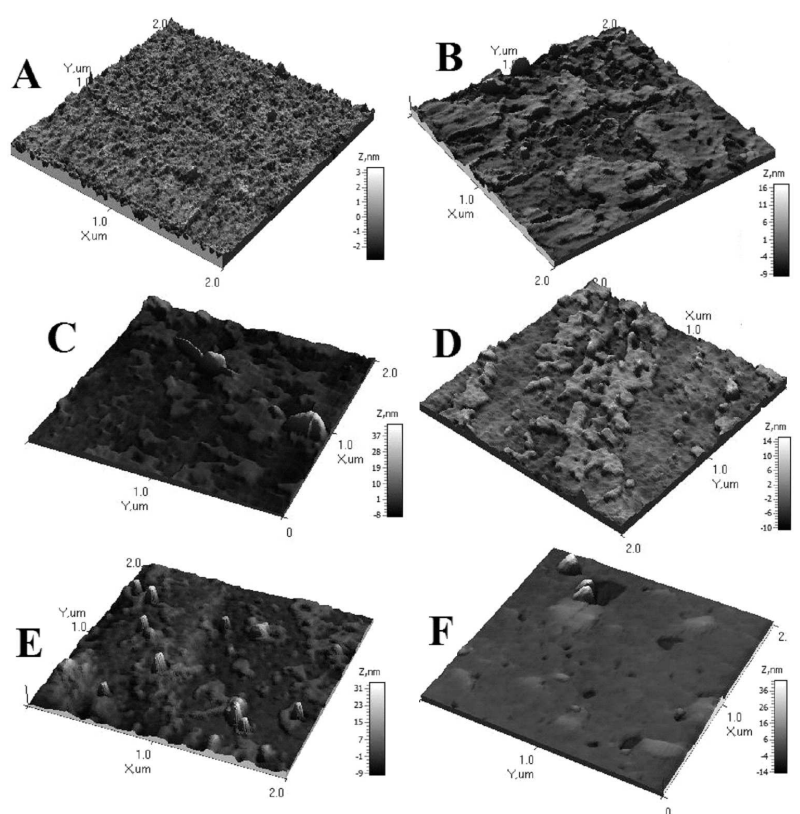

Fig. 2. AFM-images of initial glass (A) and Mo coating on glass obtained at $1.2 \times 10^{16}(\mathrm{~B}), 3.2 \times 10^{16}(\mathrm{C})$, $5.2 \times 10^{16}(\mathrm{D}), 8.1 \times 10^{16}(\mathrm{E}), 1.1 \times 10^{17}(\mathrm{~F})$ ions $/ \mathrm{cm}^{2}$.
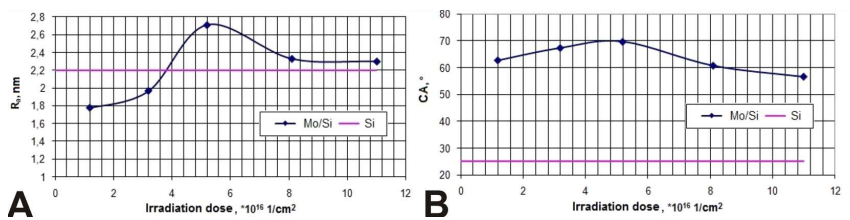

Fig. 3. Evolution of $R_{\mathrm{a}}(\mathrm{A})$ and $\Theta(\mathrm{B})$ vs. the irradiation dose for Mo/glass systems and values of $R_{\mathrm{a}}$ and $\Theta$ of initial glass.

different parts of the surface in the initial stage of the coating formation. This is evidenced, in particular, reducing the difference of heights ( $\Delta h$, Table) from $7.2 \mathrm{~nm}$ to $3 \mathrm{~nm}$ with the increasing irradiation dose and thus the deposition time.

Suvorov et al. [5] reported the presence of certain threshold values of the irradiation dose for each type of ions, the excess of which leads to the decrease of $R_{\mathrm{a}}$ of the surface, or at least, its constancy.

The average roughness of initial glass is $2.2 \mathrm{~nm}$. The AFM studies revealed the presence of irregularly distributed valleys and hills on the surface of glass (Fig. 2A). The dependence of $R_{\mathrm{a}}$ on the irradiation dose is similar to the results obtained previously [6]. Dependence of $R_{\mathrm{a}}$ on the irradiation dose is indicated in Fig. 3A. Contrary to the initial sample, the number of small irregularities is significantly reduced and the surface becomes smoother. The $R_{\mathrm{a}}$ increases with the increase of the irradiation dose to $2.71 \mathrm{~nm}$ due to the difference of levels of coverage and the multitude of islands of covering nucleation. With further increase of the deposition time, the coverage area 
increases and the surface becomes smoother which reduces the roughness to $2.3 \mathrm{~nm}$. These results indicate the possibility of managing the roughness of the film surface by changing the dose during irradiation, as reported earlier by some authors $[7,8]$.

According to the qualitative description of thin films evolution synthesized on different substrates [9] the following steps in the process of film growth: island growth, impingement and coalescence of islands, development of a continuous structure are observed.

\subsection{Wettability test}

Figure 4 shows the position of a water drop on the surface of the initial glass and Mo films on glass. Mean values of $\mathrm{CA}$ in five different positions were recorded and averaged in Table. In Fig. 3B the variation of samples $\mathrm{CA}$ with the irradiation dose is given.

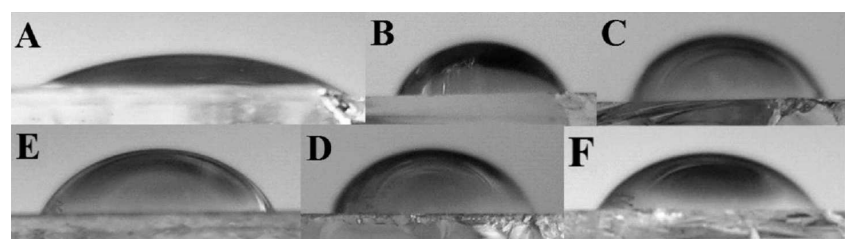

Fig. 4. Water drops on the surface of initial glass (A) and Mo coating on glass obtained at $1.2 \times 10^{16}(\mathrm{~B})$, $3.2 \times 10^{16}(\mathrm{C}), 5.2 \times 10^{16}(\mathrm{D}), 8.1 \times 10^{16}(\mathrm{E}), 1.1 \times 10^{17}$ (F) ions $/ \mathrm{cm}^{2}$.

The wettability test results show the fundamental difference between the $\Theta$ of initial glass $\left(25.1^{\circ}\right)$ and the $\Theta$ of experimentally modified surfaces $\left(56.7^{\circ}-69.7^{\circ}\right)$. The increase in the $\Theta$ by $2.45-2.77$ times when the Mo thin film is deposited on glass is observed. Thus, Mo coating deposition on glass makes the surface less hydrophilic. The dependence of the CA value on a dose of ion irradiation is similar to that of the $R_{\mathrm{a}}$ on a dose. Three factors affect the surface wettability: chemical composition of the material (deposition of Mo film), local inhomogeneity (\% of coating formation) and surface morphology (surface roughness). These results indicate the possibility of managing the water wettability of the Mo thin film on glass by changing the dose during irradiation. The results can be also useful for obtaining a surface with desirable wettability in applications not related to solar energy. Both hydrophobic and hydrophilic surfaces have been extensively investigated due to their importance for industrial applications.

\section{Conclusions}

Investigations of influence of Mo deposition on glass substrates by SIAD, on its surface topography and wet- tability were carried out. In the paper some steps in the process of film growth are presented.

It was found out that with the increase of the irradiation dose, the average roughness increases rapidly at first and then decreases. The threshold value of irradiation dose excess which leads to surface roughness decrease is $5.2 \times 10^{16}$ ions $/ \mathrm{cm}^{2}$. It has been also qualitatively estimated that coating is harder than a substrate.

Contact angle measurements showed that the deposition of the Mo thin film on glass makes the surface less hydrophilic. The increase $(2.45-2.77)$ times in the contact angle of water when the Mo thin film is deposited on glass is observed. The dependence of the $\mathrm{CA}$ value on a dose is similar to that of $R_{\mathrm{a}}$ on a dose. It was found out that deposition of Mo thin film on glass with different irradiation doses can be used for obtaining a surface of desirable topography and water wettability.

\section{Acknowledgments}

This research has been partly supported by Belarusian Republican Foundation for Fundamental Research project $\mathrm{F} 11 \mathrm{OB}-028$.

\section{References}

[1] K. Orgassa, H.W. Schock, J.H. Werner, Thin Solid Films 431-432, 387 (2003).

[2] V.V. Uglov, V.M. Anishchik, V.M. Astashynski, N.N. Cherenda, G.I. Gimro, A.V. Kovyazo, Surf. Coat. Technol. 200, 245 (2005).

[3] I.S. Tashlykov, O.G. Bobrovich, V.Ch. Palchekh, V.V. Tuljev, N.V. Alov, V.S. Kulikauskas, G.K. Wolf, Surf. Coat. Technol. 74-75, 945 (1995).

[4] I.S. Tashlykov, A.V. Kasperovich, G.K. Wolf, Surf. Coat. Technol. 158-159, 498 (2002).

[5] A.L. Suvorov, Yu.N. Cheblukov, N.E. Lazarev, A.F. Bobkov, M.O. Popov, V.P. Babaev, Techn. Phys. 45, 343 (2000).

[6] I.S. Tashlykov, A.I. Turavets, P. Zukowski, Acta Phys. Pol. A 120, 115 (2011).

[7] Z. Li, D. Chen, X. Fu, W. Miao, Z. Zhang, Adv. Mater. Sci. Eng. 2012, 640462 (2012).

[8] A.V. Merkulov, O.A. Merkulova, Techn. Phys. 44, 230 (1999).

[9] I. Petrov, P.B. Barna, L. Hultman, J. Greene, J. Vac. Sci. Technol. A 21, S117 (2003). 